\title{
Heterologous expression of PvACR3;1 decreased arsenic accumulation in plant shoots
}

\author{
Y.S. Chen, Y. Cao, C.Y. Hua, M.R. Jia, J.W. Fu, Y.H. Han, X. Liu \& L.Q. Ma \\ State Key Lab of Pollution Control and Resource Reuse, School of the Environment, \\ Nanjing University, Jiangsu, P.R. China
}

\begin{abstract}
Arsenic (As) is a toxic carcinogen so it is crucial to decrease As accumulation in crops to reduce its risk to human health. Arsenite (As(III)) antiporter ACR3 protein is critical for As metabolism in organisms, but it is lost in flowering plants. Here, a novel ACR3 gene from As hyperaccumulator Pteris vittata, PvACR3; 1, was cloned and expressed in Saccharomyces cerevisiae (yeast), Arabidopsis thaliana (model plant), and Nicotiana tabacum (tobacco). Yeast experiments showed that PvACR3;1 functioned as an As(III)-antiporter to mediate As(III) efflux to an external medium. At $5 \mu \mathrm{M}$ As(III), PvACR3;1 transgenic Arabidopsis accumulated 14-29\% higher As in the roots and 55-61\% lower As in the shoots compared to WT control, showing lower As translocation. Besides, transgenic tobacco under $5 \mu \mathrm{M}$ As(III) or As(V) (arsenate) also showed similar results, indicating that expressing PvACR3;1 gene increased As retention in plant roots. Moreover, observation of PvACR3;1-green fluorescent protein fusions in transgenic Arabidopsis showed that PvACR3;1 protein localized to the vacuolar membrane, indicating that PvACR3;1 mediated As(III) sequestration into vacuoles, consistent with increased root As. Thus, our study provides a potential strategy to limit As accumulation in plant shoots, shedding light on engineering low-As crops to improve food safety.
\end{abstract}

\section{INTRODUCTION}

In yeast, As(III) transporter ACR3 (Arsenic Compounds Resistance 3 ) is localized to the plasma membrane to export As(III) out of the cell (Wysocki et al., 1997). Its homologues exist in plants including moss, lycophytes, ferns, and gymnosperms, but not angiosperms (Indriolo et al., 2010). In Ashyperaccumulator Pteris vittata, two ACR3 homologues, PvACR3 and PvACR3;1, were reported, with PvACR3 being localized to the vacuolar membrane and likely efflux of As(III) into the vacuole for sequestration in $P$. vittata (Indriolo et al., 2010). However, in transgenic Arabidopsis, PvACR3 localizes to the plasma membrane and its heterologous expression increases As(III) efflux and As(III) translocation (Chen et al., 2013; Wang et al., 2017). Although PvACR3;1 was reported by Indriolo et al. (2010), it was not investigated in their study so its function is unclear. Here, in this study, we successfully cloned the PvACR3;1 full length coding sequence (CDS) from $P$. vittata and tested its function following expression in transgenic yeast and in transgenic plants.

\section{METHODS/EXPERIMENTAL}

\subsection{Yeast growth assays}

PvACR3;1 CDS was cloned into the GAL1 promoter cassette of yeast vector pAG413GAL-ccdB by recombination. Then it was transformed into $\Delta a c r 3$ mutant with BY4741 background. Yeast transformants that expressing PvACR3;1 were then selected for growth assays.

\subsection{Transgenic plant generation, plant growth and As determination}

PvACR3;1 CDS was cloned into the CaMV 35S promoter cassette of plant expression vector pSN1301 by recombination. Agrobacterium strain C58 was transformed with the binary vector by electroporation. The Agrobacterium culture was used to transform Arabidopsis thaliana and tobacco, respectively. For analysis of As accumulation in transgenic plants, plants were cultivated under different $\mathrm{As}(\mathrm{III})$ or $\mathrm{As}(\mathrm{V})$ treatments. Total As concentrations were determined by inductively coupled plasma mass spectrometry (ICP-MS).

\section{RESULTS AND DISCUSSION}

\subsection{PvACR3; 1 encodes a functional As(III) antiporter in yeast}

Expression of PvACR3;1 enhanced the tolerance of $\Delta a c r 3$ to both $\mathrm{As}(\mathrm{III})$ and $\mathrm{As}(\mathrm{V})$ and effectively suppressed As-sensitive phenotype. Arsenic determination showed that expressing PvACR3;1 significantly reduced As accumulation in the yeast after growing under $\mathrm{As}(\mathrm{III})$ or $\mathrm{As}(\mathrm{V})$. Considering $\mathrm{As}(\mathrm{V})$ can be reduced to $\mathrm{As}(\mathrm{III})$ in yeast cells, we concluded 

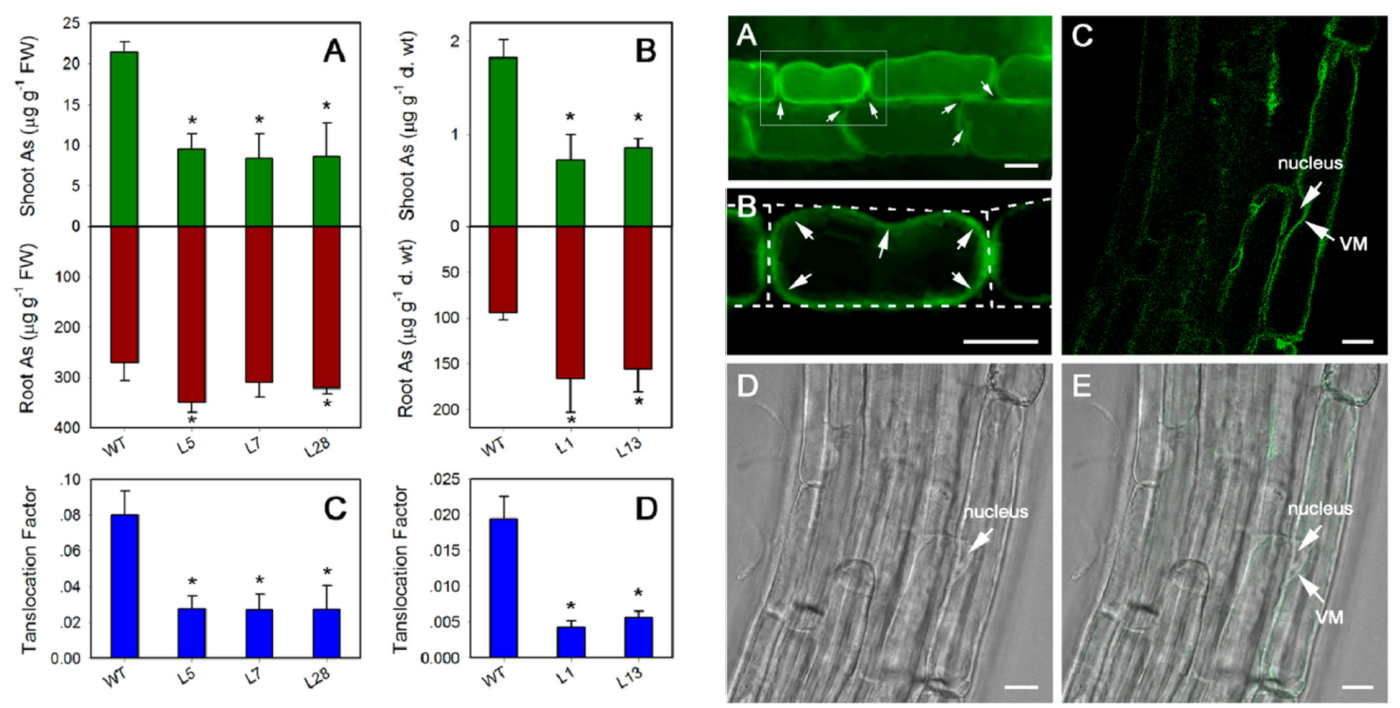

Figure 1. Arsenic (As) accumulation and translocation in PvACR3;1 transgenic plants under $5 \mu \mathrm{M}$ arsenite (As(III)) treatments.

that the obtained PvACR3;1 was a functional As(III) antiporter, which played an important role in As(III) efflux to the external medium across the yeast plasma membrane.

\subsection{Expressing PvACR3; 1 decreased shoot As accumulation in plants under As(III) exposure}

PvACR3;1 gene was transformed into model plant Arabidopsis and tobacco and three transgenic Arabidopsis lines (L5, L7, and L28) and two transgenic tobacco lines (L1 and L13) were obtained. At $5 \mu \mathrm{M}$ As(III), both transgenic Arabidopsis and transgenic tobacco lines accumulated higher As in the roots and lower As in the shoots, compared to the WT controls (Fig. 1A,B). As a result, As translocation dropped by $\sim 66 \%$ in transgenic Arabidopsis L5, L7, and L28 and by $71-78 \%$ in transgenic tobacco L1 and L13, compared to WT controls (Fig. 1C,D). These results showed that heterologous expression of PvACR3;1 decreased As(III) translocation in plants, probably by retaining As(III) in plant roots.

\subsection{Subcellular localization of PvACR3;1 in plants}

The subcellular location of PvACR3;1 in plants is critical for its physiological function. Thus, vector expressing (C-terminal) PvACR3;1-GFP fusions was transformed into transgenic Arabidopsis to visualize GFP fluorescence. GFP signal of PvACR3;1-GFP showed a clear localization of GFP to the indented region of the rectangular cell (Fig. 2A,B). In addition, the PvACR3;1-GFP also showed a clear localization of GFP delineating the nucleus at the root cell, unambiguously demonstrating a vacuolar location (Fig. 2C-E), indicating that PvACR3;1 may mediate As(III) sequestration in transgenic plant roots, thus increasing As accumulation in the roots and subsequently decreasing

Figure 2. Subcellular localization of PvACR3;1 in plants through the observation of GFP fluorescence of PvACR3;1-GFP fusions.

As(III) xylem loading and As translocation to plant shoots.

\section{CONCLUSIONS}

PvACR3;1 localized to vacuolar membrane when heterologous expressed in plants and mediated As(III) sequestration into vacuoles in the roots, therefore reducing As accumulation in plant shoots. Thus, our work provides a potential strategy to decrease As accumulation in plant shoots for food safety.

\section{ACKNOWLEDGEMENTS}

This work was supported by Jiangsu Provincial Natural Science Foundation (Grant No. BK20160649), and the National Natural Science Foundation of China (Grant No. 21707068, 21637002).

\section{REFERENCES}

Chen, Y., Xu, W., Shen, H., Yan, H., He, Z. \& Ma, M. 2013. Engineering arsenic tolerance and hyperaccumulation in plants for phytoremediation by a $P v A C R 3$ transgenic approach. Environ. Sci. Technol. 47(16): 9355-9362.

Indriolo, E., Na, G., Ellis, D., Salt, D.E. \& Banks, J.A. 2010. A vacuolar arsenite transporter necessary for arsenic tolerance in the arsenic hyperaccumulating fern Pteris vittata is missing in flowering plants. Plant Cell 22(6): 2045-2057.

Wang, C., Na, G., Bermejo, E.S., Chen, Y., Banks, J.A., Salt, D.E. \& Zhao, F.J. 2018. Dissecting the components controlling root-to-shoot arsenic translocation in Arabidopsis thaliana. New Phytol. 217(1):206-218.

Wysocki, R., Bobrowicz, P. \& Ulaszewski, S. 1997. The Saccharomyces cerevisiae ACR3 gene encodes a putative membrane protein involved in arsenite transport. J. Biol. Chem. 272(48): 30061-30066. 